\title{
Emergency Department Revisits Due to Cast-Related Pain in Children with Forearm Fractures
}

This article was published in the following Dove Press journal:

Journal of Pain Research

\author{
Noa Kvatinsky' \\ Rivka Carmiel $\mathbb{( D}^{2}$ \\ Ronit Leiba ${ }^{3}$ \\ Itai Shavit ${ }^{1}$
}

'Pediatric Emergency Department, Rambam Health Care Campus, Haifa, Israel; ${ }^{2}$ Rappaport Faculty of Medicine, Technion-Israel Institute of Technology, Haifa, Israel; ${ }^{3}$ Quality of Care Unit, Rambam Health Care Campus, Haifa, Israel
Correspondence: Itai Shavit

POB 274, Kibutz Maayan Tzvi, Haifa

3080500 , Israel

Tel +972-50-2063239

Fax +972-4-777-4012

Email itai@pem-database.org
Background: Cast immobilization is the primary treatment for children with forearm fractures. After emergency department (ED) discharge, some patients develop cast-related pain (CstRP) around the distal part of the upper extremity. We examined variables associated with ED revisits due to CstRP in children with forearm fractures.

Methods: A retrospective cohort study of all children who were treated with cast immobilization for forearm fracture over a 7-year period was conducted. Patient demographics, fracture location, casting method (below elbow/above elbow), first visit pain scores, treatment with fracture reduction, and revisit data were collected. Multivariate regression was used to identify predictors of revisits due to CstRP within $72 \mathrm{hrs}$ post-discharge.

Results: A total of 2307 children were treated with cast immobilization; 95 (4.2\%) revisited the ED due to CstRP (median pain score 7, interquartile range 5-9). No patient had neurovascular compromise or required surgery or re-reduction. Fifty-eight $(61.1 \%)$ patients were treated with cast splitting, $10(10.5 \%)$ with trimming, and 27 (28.4\%) with cast replacement. Variables on first visit that were associated with increased odds for ED revisit included treatment with fracture reduction (odds ratio [OR] 2.31; 95\% confidence interval [CI] 1.58-3.36) and a median pain score of 6 or more upon ED presentation (OR 1.57; 95\% CI 1.32-2.13).

Discussion: A small number of children with forearm fractures revisited the ED due to CstRP. Study findings suggest that being treated with closed reduction and having a pain score $\geq 6$ on the first visit were associated with ED revisit due to CstRP.

Keywords: child, fracture, casting, pain

\section{Introduction}

Forearm fracture is the most common type of fracture in children presenting to the emergency department (ED) and composes approximately one-quarter of all fractures in childhood. ${ }^{1}$ Cast immobilization remains the primary treatment for patients with forearm fractures. However, approximately $6 \%$ of the patients return unexpectedly to the ED due to complications related to the cast. ${ }^{2,3}$ Some patients return because of a damaged cast (e.g. wet cast, loose cast), while others present with symptoms such as swelling, color change, skin irritation or pain around the distal part of the upper extremity (cast-related pain, CstRP). ${ }^{3-6}$ Patients who have pain need to be urgently evaluated to rule out neurovascular deficit and are treated with analgesics, cast splitting, trimming, or replacement. ${ }^{7}$

There is a paucity of literature on factors contributing to ED revisit due to CstRP in children with forearm fractures. Understanding the factors associated with ED revisits due to CstRP may help to predict which patients are more likely to develop CstRP. 
We sought to examine variables associated with ED revisits due to CstRP in children with forearm fractures.

\section{Patients and Methods}

\section{Study Setting, Design, and Subjects}

The study was conducted at a level-one trauma center that serves a population of approximately 600,000 children. A retrospective cohort study was conducted over a period of 7 years (01 January 2012 to 31 December 2018). The electronic medical records of all ED patients aged 3 to 18 years who were diagnosed with non-torus forearm fractures were extracted and analyzed. The patient records and information were anonymized and deidentified before analysis. The Institutional Review Board of Rambam Health Care Campus (No. 523-17) approved this study without the requirement of obtaining informed consent.

\section{ED Pain Protocol}

Three self-reported instruments are used by the ED nursing staff to assess pain in triage at the age range of 3 to 18 years: the Wong-Baker rating scale for children aged 3 to 7 years, the Visual Analog Scale (VAS) for children aged 8 to 14 years, and the Numeric Rating Scale (NRS) for children 15-18 years (Figure 1). ${ }^{8}$ Children who report their pain in triage as $1-3$ receive oral acetaminophen $15 \mathrm{mg} / \mathrm{kg}$ (maximum of $650 \mathrm{mg}$ ). Children who report their pain as 4-6 receive oral dipyrone $15 \mathrm{mg} / \mathrm{kg}$, and children who report their pain as $7-10$ receive oral morphine $0.2 \mathrm{mg} / \mathrm{kg}$, oral oxycodone $0.2 \mathrm{mg} / \mathrm{kg}$, or intranasal fentanyl $1.5 \mathrm{mcg} / \mathrm{kg}$. ${ }^{9,10}$ According to department protocol, pain level in children with fractures is reassessed before discharge to verify that patients are discharged with a pain score of 4 or less. At discharge, caregivers are provided with an information pamphlet by the patient's nurse. They are instructed to use ibuprofen $(10 \mathrm{mg} / \mathrm{kg}$, max. $600 \mathrm{mg})$ every $6 \mathrm{hrs}$ as needed for pain for $48 \mathrm{hrs}$ after discharge, and acetaminophen $15 \mathrm{mg} / \mathrm{kg}$ if pain arises between doses of ibuprofen. ${ }^{11}$

\section{Study Outcome Measure}

The outcome measure of the study was CstRP, which was defined as the presence of pain related to the cast around the distal part of the upper extremity.

\section{PAIN ASSESSMENT}

Wong-Baker FACES Pain Rating Scale: 3-7 years old

Visual Analog Scale: 8-15 years old

Numeric Rating Scale: $16-18$ years old

Mild pain (1-3)

Paracetamol $-15 \mathrm{mg} / \mathrm{kg}$

orally or intravenously
Moderate pain (4-6)

Oral Dipyrone $-15 \mathrm{mg} / \mathrm{kg}$

Oral Ibuprofen $-10 \mathrm{mg} / \mathrm{kg}$
Severe pain (7-10)

Oral Oxycodone $-0.2 \mathrm{mg} / \mathrm{kg}$

Oral Morphine $-0.2 \mathrm{mg} / \mathrm{kg}$

Intranasal Fentanyl $-1.5 \mathrm{mcg} / \mathrm{kg}$

- Collar-and-cuff sling for children suspected of having non-displaced fractures

- Temporary splint and collar-and cuff sling for children suspected of having displaced fractures

Figure I Emergency department protocol for fracture pain assessment and management at the age range of 3-18 years. 


\section{ED Casting Protocol}

Non-torus forearm fractures are treated with a circumferential plaster cast by an orthopedic surgeon. The same method of casting is practiced by all orthopedic surgeons, including attending physicians and residents. Previous studies demonstrated that short arm (below-theelbow) casts perform as well as long arm (above-the-elbow) casts for maintaining the reduction of fractures in the distal third of the forearm in children, with a similar rate of complications. However, in children under the age of 4 years, an above-elbow cast is still recommended as short arm casts may slip. ${ }^{11,12}$ In our institution, patients with distal forearm fractures who are older than 4 years, a short arm (below elbow) cast is applied, while patients younger than 4 years are treated with a long arm (above elbow) cast. According to department protocol, children with displaced forearm fractures are treated with closed reduction performed by the orthopedic surgeon. The procedure is performed under deep sedation provided by an emergency physician. ${ }^{13}$ Patients with fractures in an acceptable radiologic alignment after reduction are discharged and instructed to return to the orthopedic clinic within 5-7 days or to the ED if they experience CstRP. Prior to ED discharge, patients and caregivers receive an explanation about the possibility of CstRP and are asked to return to the ED for reevaluation if CstRP or other symptoms of neurovascular injury present. Patients returning to the ED with such symptoms are examined by an orthopedic surgeon and may be treated with analgesia, cast splitting, trimming, or complete replacement to relieve symptoms. ${ }^{7}$

\section{Data Collection}

The International Classification of Disease - 9th Revision (ICD-9) codes were used to identify patients who were discharged from the pediatric ED with the diagnosis of forearm fracture between 01 January 2012 and 31 December 2018. Patients younger than 3 years of age, patients with torus fractures (treated with splinting rather than casting), patients with open fractures, and patients who were treated in the operating room were excluded.

Patient's records were available from the ED electronic medical records system ("Prometheus integrated computer system"). The Prometheus integrated computer system was developed by the hospital's Department of Information Technology and is a mandatory working tool for physicians and nursing staff and includes nurses and physicians full report. ${ }^{14}$
The following variables were extracted from the electronic registry: age, gender, location of fracture (distal radius, mid-shaft/proximal shaft radius, radius and ulna), type of casting (below elbow, above elbow), pain score on admission to the ED, pain score prior to ED discharge, treatment with fracture reduction in the ED (yes/no), return ED visit due to CstRP (yes/no). For returned patients, the following variables were also extracted: pain score on ED admission on return visit, patient's/caregiver's complaint (damaged cast, pain/discomfort around the extremity/fingers, swelling, color change, skin irritation), any treatment on return visit, admission to the hospital for pain management (yes/no). Files were reviewed independently by two abstractors (RC and NK) using a data abstraction instrument. The two abstractors underwent standardized training to perform chart review on the study population using the electronic health records of the hospital. Chart reviews were conducted in accordance with published methods for retrospective chart review studies. ${ }^{15}$

\section{Statistical Analysis}

Multivariable logistic regression was performed to identify variables associated with ED revisits due to CstRP in children with non-torus forearm fractures. Variables with a $p$ value $<0.10$ on univariate analysis as well as variables with a biologically plausible relationship were considered for inclusion in a regression model. The potential covariates included age, gender, locations of fracture (distal radius, mid-shaft/proximal shaft radius, radius and ulna), type of casting (below elbow, above elbow), pain score on admission to the first ED visit, undergoing fracture reduction in first ED visit. Data were analyzed with SPSS 21 version (SPSS-IBM, Chicago, IL).

\section{Results}

A total of 2307 children with non-torus forearm fractures with a mean age of $9.6 \pm 3.8$ years and a male/female ratio of 1684 / 623 were treated with cast immobilization during the 7-year study period. Cast immobilizations were performed by 71 orthopedic surgeons. One hundred and fifty-three/2307 (6.7\%) patients revisited the ED due to problems related to the cast, 95/2307 (4.2\%) of them were painful (Figure 2). Median pain score was 7 (interquartile range 5-9). Patients were treated with a single dose of dipyrone, ibuprofen, oral morphine or oral oxycodone. There were no cases of neurovascular compromise, no patient required surgery or rereduction, and no patient was admitted to the hospital for pain management. Fifty-eight/95 (61.1\%) patients were 


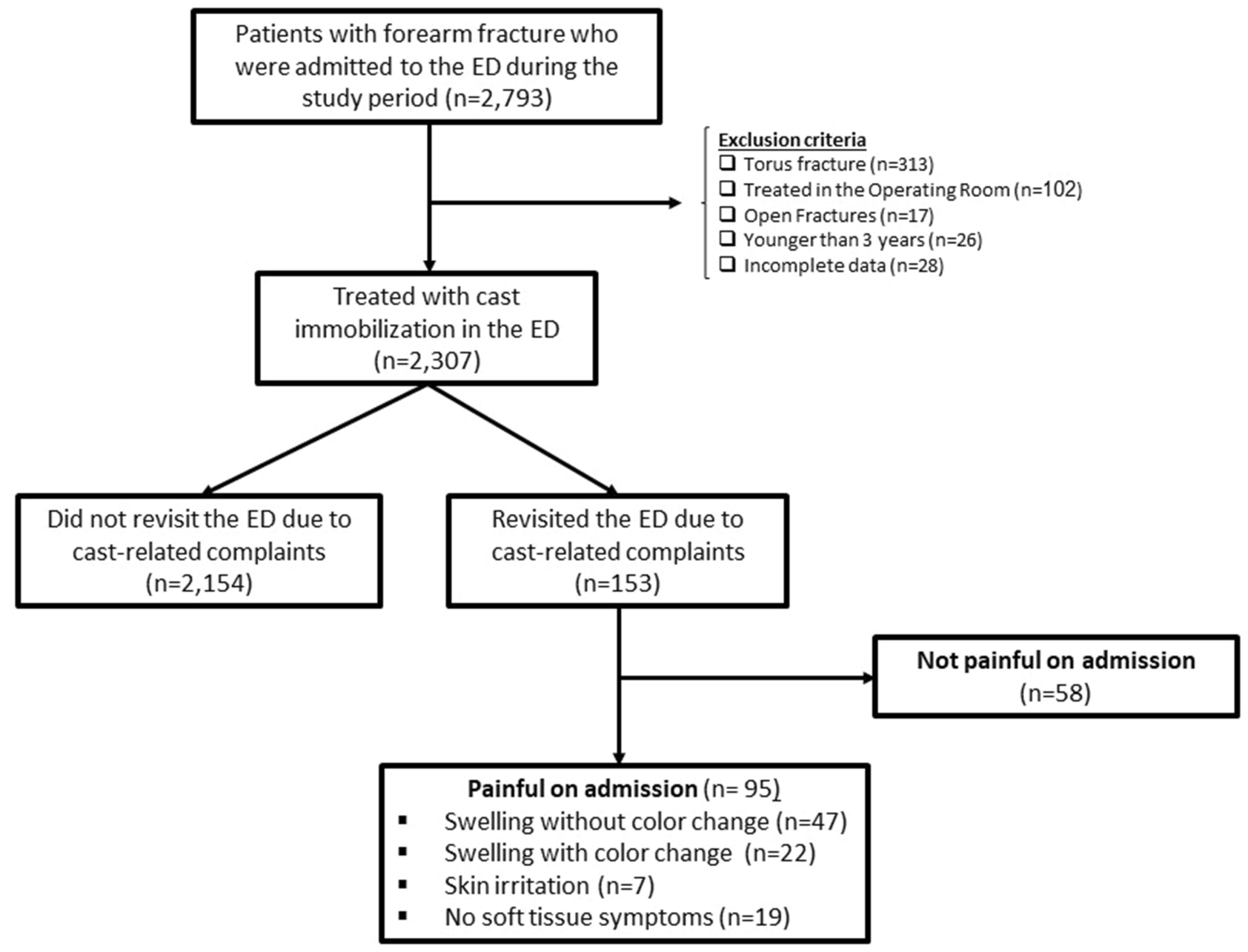

Figure 2 Study flowchart.

Abbreviation: ED, Emergency Department.

treated with cast splitting, 10/95 (10.5\%) with trimming, and $27 / 95(28.4 \%)$ with cast replacement. Table 1 presents the demographic characteristics of the 95 patients who had ED revisits due to CstRP.

Variables that were mostly associated with increased odds for revisit due to CstRP included fracture reduction on first visit [odds ratio $(\mathrm{OR})=2.31,95 \%$ confidence interval (CI): $1.58-3.36]$ and median pain score of 6 or more [OR $=1.57,95 \% \mathrm{CI}: 1.32-2.13]$ (Table 2).

\section{Discussion}

The main finding of our study is that a certain amount (4.2\%) of children with forearm fracture revisited the ED due to CstRP. In our cohort, two variables were mostly associated with ED revisit due to CstRP; being treated with closed reduction, and having a pain score of 6 or more on ED admission. Age, gender, type of cast immobilization (short cast or long cast) and location of the fracture (distal radius, mid-shaft/proximal shaft radius, radius and ulna) were not associated with revisits due to CstRP. These findings suggest that physicians should be more proactive in discussing the potential risk for CstRP in patients who underwent fracture reduction and patients who had significant pain level on ED admission. A possible way of preventing CstRP is splitting the cast, a method that many surgeons advocate. Previous studies reported the benefit of splitting or spreading of fiberglass casts. ${ }^{16,17}$ It is worth mentioning that a randomized controlled study failed to show any difference in the incidence of cast-related problems between closed cast and split cast in patients with forearm fractures. ${ }^{18}$

The returned patients had a median pain score of 7 and were treated with analgesics according to our pain protocol. This finding implies that CstRP could lead to a severe pain that justifies early analgesia. In our study, two-thirds (95/153) of the patients who revisited the ED due to cast related complaints, were painful. Sawyer et al reported that only 
Table I Demographic Characteristics of Children Who Had an Emergency Department Revisit Due to Cast-Related Pain ( $n=95)$

\begin{tabular}{|l|l|}
\hline $\begin{array}{l}\text { Age, mean } \pm \text { SD } \\
\text { Males/Females }\end{array}$ & $\begin{array}{l}10.2 \pm 3.8 \\
116 / 37\end{array}$ \\
\hline $\begin{array}{l}\text { Fracture type } \\
\text { Radius and Ulna, } \mathrm{n}(\%)\end{array}$ & $75(49 \%)$ \\
$\begin{array}{l}\text { Distal Radius, } \mathrm{n}(\%) \\
\text { Mid-shaft or Proximal Shaft of Radius, } \mathrm{n}(\%)\end{array}$ & $6(47 \%)$ \\
\hline $\begin{array}{l}\text { Below elbow cast/Above elbow cast } \\
\text { Treated with fracture reduction, } \mathrm{n}(\%)\end{array}$ & $122 / 3 \mathrm{I}$ \\
Pain score on admission on first ED visit, median (IQR) & $\begin{array}{l}80(52.5 \%) \\
6(4-8)\end{array}$ \\
\hline
\end{tabular}

Abbreviations: SD, Standard Deviation; IQR, Interquartile Range; ED, Emergency Department; CstRP, Cast-Related Pain.

Table 2 Results of Multivariate Regression of Predictors of ED Revisit Due to Cast-Related Pain $(n=2307)$

\begin{tabular}{|c|c|c|}
\hline Predictor & $\begin{array}{l}\text { Odds } \\
\text { Ratio }\end{array}$ & $95 \% \mathrm{Cl}$ \\
\hline $\begin{array}{l}\text { Older age } \\
\text { Female gender }\end{array}$ & $\begin{array}{l}1.05 \\
0.86\end{array}$ & $\begin{array}{l}0.99-1.10 \\
0.68-2.04\end{array}$ \\
\hline $\begin{array}{l}\text { Pain score }<6 \text { on admission to the first ED } \\
\text { visit } \\
\text { Pain score } \geq 6 \text { on admission to the first ED } \\
\text { visit }\end{array}$ & $\begin{array}{l}\text { Ref } \\
1.57\end{array}$ & $\begin{array}{l}- \\
1.32-2.13\end{array}$ \\
\hline $\begin{array}{l}\text { Fracture location } \\
\text { Distal radius } \\
\text { Mid-shaft/proximal shaft radius } \\
\text { Radius and ulna }\end{array}$ & $\begin{array}{l}\text { Ref } \\
0.98 \\
1.16\end{array}$ & $\begin{array}{l}- \\
0.89-3.41 \\
0.92-2.56\end{array}$ \\
\hline $\begin{array}{l}\text { No treatment with fracture reduction on } \\
\text { first ED visit } \\
\text { Treatment with fracture reduction on first } \\
\text { ED visit }\end{array}$ & $\begin{array}{l}\text { Ref } \\
2.31\end{array}$ & $\begin{array}{l}- \\
1.58-3.36\end{array}$ \\
\hline $\begin{array}{l}\text { Type of casting } \\
\text { Below elbow } \\
\text { Above elbow }\end{array}$ & $\begin{array}{l}\text { Ref } \\
1.02\end{array}$ & $\begin{array}{l}- \\
0.69-3.71\end{array}$ \\
\hline
\end{tabular}

Abbreviations: $\mathrm{Cl}$, Confidence Interval; ED, Emergency Department.

$10 \%$ of the patients with cast-related complications present with pain. ${ }^{3}$ We believe that our results better reflect the real situation because we abstracted the data from the triage process in which pain is assessed using standardized pain assessment protocol tools (Figure 1). ${ }^{8}$ Another important finding is that despite the fact that all the 95 painful patients were treated with cast splitting, trimming or replacement, no case of neurovascular compromise was recorded and no patient required surgery, re-reduction, or hospital admission for pain management. These results suggest that in children CstRP is infrequently associated with neurological deficit.
Several other pediatric studies corroborate our findings. ${ }^{3-5}$ It is worth mentioning, however, that most of the children with CstRP needed cast work to potentially prevent a compartment syndrome. In adults, neuropathic symptoms were reported in $6 / 296(2 \%)$ of the patients with forearm fracture. None of the patients had carpel tunnel syndrome or compartment syndrome. One patient had decreased sensation over the ulnar nerve distribution. ${ }^{7}$

This study has certain limitations. Firstly, it has limitations inherent in a retrospective chart review, including dependence on the quality of documentation. As the information extracted from the medical records was objective and performed by two researchers, independently, we believe that our study was not subject to abstractor bias. Secondly, our data did not allow us to distinguish between displaced fractures and non-displaced fractures because this information was not always recorded in the charts. Thirdly, this study is a single-center study and, although the epidemiological data are comparable to those of earlier studies, we do not know whether our results apply to the general population. Fourthly, patients were instructed to return to our ED if they had CstRP; however, it is possible that some patients returned to their family physician or to another ED in the region. It is therefore possible that the actual revisit rate is higher than the $4.2 \%$ found in our study.

In conclusion, we found that a small number of children with forearm fractures revisited the ED due to CstRP. Study findings suggest that being treated with closed reduction and having a pain score $\geq 6$ on the first visit were associated with ED revisit due to CstRP.

\section{Abbreviations}

ED, Emergency Department; CstRP, Cast-Related Pain.

\section{Acknowledgments}

An abstract of this paper was presented at the February 2019 annual conference of the Israeli Society for Clinical Pediatrics as a poster presentation with interim findings. The poster's abstract was published in the proceedings of the conference.

\section{Author Contributions}

Noa Kvatinsky and Rivka Carmiel collected and interpreted the data, carried out the initial analysis, reviewed the literature, and critically revised the article. Rivka Carmiel and Noa Kvatinsky have equal contributions. Ronit Leiba carried out the statistical analysis, reviewed the literature, and critically revised the article. Itai Shavit conceived the idea for the 
study, designed the study, analyzed the data, and drafted the manuscript. All authors are responsible for the reported research, approved the manuscript as submitted. Prof. Shavit has full access to all the data in the study and had final responsibility for the decision to submit for publication. All authors contributed towards data analysis, drafting and critically revising the paper, gave final approval ofthe version to be published, and agreed to be accountable for all aspects of the work.

\section{Disclosure}

For all 4 authors, there were no potential conflicts of interest, real or perceived in the study design, the collection, analysis, and interpretation of data, the writing of the report, and the decision to submit the paper for publication. No honorarium, grant, or other form of payment was given to anyone to produce the manuscript. The authors have no conflicts of interest to declare.

\section{References}

1. Ryan LM, Teach SJ, Searcy K, et al. Epidemiology of pediatric forearm fractures in Washington, DC. J Trauma. 2010;69:s200-s201. doi:10.1097/TA.0b013e3181f1e837

2. Jiang N, Cao ZH, Ma YF, et al. Management of pediatric forearm torus fractures: a systematic review and meta-analysis. Pediatr Emerg Care. 2016;32:773-778. doi:10.1097/PEC.0000000000000579

3. Sawyer JR, Ivie CB, Huff AL, et al. Emergency room visits by pediatric fracture patients treated with cast immobilization. J Pediatr Orthop. 2010;30:248-252. doi:10.1097/BPO.0b013e3181d213bc

4. Difazio RL, Harris M, Feldman L, et al. Reducing the incidence of cast-related skin complications in children treated with cast immobilization. J Pediatr Orthop. 2017;37:526-531. doi:10.1097/B PO.0000000000000713

5. Halanski M, Noonan KJ. Cast and splint immobilization: complications. $J$ Am Acad Orthop Surg. 2008;16:30-40. doi:10.5435/00124635200801000-00005

6. Khan S, Sawyer J, Pershad J. Closed reduction of distal forearm fractures by pediatric emergency physicians. Acad Emerg Med. 2010;17:1169-1174. doi:10.1111/acem.2010.17.issue-11
7. Drager J, Carli A, Matache BA, Harvey EJ. Circumferential casting of distal radius fractures. J Orthop Trauma. 2014;28:e186-e190. doi:10.1097/BOT.0000000000000045

8. Shavit I, Brumer E, Shavit D, et al. Emergency department pain management in pediatric patients with fracture or dislocation in a bi-ethnic population. Ann Emerg Med. 2016;67:9-14. doi:10.1016/j. annemergmed.2015.07.497

9. Friday JH, Kanegaye JT, McCaslin I, et al. Ibuprofen provides analgesia equivalent to acetaminophen-codeine in the treatment of acute pain in children with extremity injuries: a randomized clinical trial. Acad Emerg Med. 2009;16:711-716. doi:10.1111/j.15532712.2009.00471.x

10. Clark E, Plint AC, Correll R, et al. A randomized, controlled trial of acetaminophen, ibuprofen, and codeine for acute pain relief in children with musculoskeletal trauma. Pediatrics. 2007;119:460-467. doi:10.1542/peds.2006-1347

11. Giacalone M, Capua T, Shavit I. Short and long arm cast and pain after discharge in children who underwent reduction of distal forearm fracture in the Emergency Department: a study protocol for a randomized comparative effectiveness study. Contemp Clin Trials Commun. 2018;11:46-49. doi:10.1016/j.conctc.2018.06.003

12. Webb GR, Galpin RD, Armstrong DG. Comparison of short and long arm plaster casts for displaced fractures in the distal third of the forearm in children. J Bone Joint Surg Am. 2006;88(1):9-17. doi:10.2106/JBJS.E.00131

13. Scheier E, Gadot C, Leiba R, et al. Sedation with the combination of ketamine and propofol in a pediatric ed: a retrospective case series analysis. Am J Emerg Med. 2015;33:815-817. doi:10.1016/j.ajem. 2015.03.033

14. Shavit I, Feldman O. Emergency department waiting times in an Israeli children's hospital during times of military conflict. Mil Med. 2018;183:e28-e31. doi:10.1093/milmed/usx054

15. Kaji AH, Schriger D, Green S. Looking through the retrospectoscope: reducing bias in emergency medicine chart review studies. Ann Emerg Med. 2014;64:292-298. doi:10.1016/j.annemergmed.2014. 03.025

16. Kleis K, Schlechter JA, Doan JD, et al. Under pressure: the utility of spacers in univalved fiberglass casts. J Pediatr Orthop. 2019;39 (6):302-305. doi:10.1097/BPO.0000000000000961

17. Zaino CJ, Patel MR, Arief MS, et al. The effectiveness of bivalving, cast spreading, and webril cutting to reduce cast pressure in a fiberglass short arm cast. J Bone Joint Surg Am. 2015;97 (5):374-380. doi:10.2106/JBJS.N.00579

18. Schulte D, Habernig S, Zuzak T, et al. Forearm fractures in children: split opinions about splitting the cast. Eur J Pediatr Surg. 2014;24:163-167. doi:10.1055/s-0033-1341412
Journal of Pain Research

\section{Publish your work in this journal}

The Journal of Pain Research is an international, peer reviewed, open access, online journal that welcomes laboratory and clinical findings in the fields of pain research and the prevention and management of pain. Original research, reviews, symposium reports, hypothesis formation and commentaries are all considered for publication. The manuscript management system is completely online and includes a very quick and fair peer-review system, which is all easy to use. Visit http:// www.dovepress.com/testimonials.php to read real quotes from published authors. 